\title{
Recent Developments in Rodent Models of High-Fructose Diet-Induced Metabolic Syndrome: A Systematic Review
}

\author{
Alvin Man Lung Chan ${ }^{1,2}$, Angela Min Hwei Ng ${ }^{1}$, Mohd Heikal Mohd Yunus ${ }^{3}{ }^{\circledR}$, Ruszymah Bt Hj Idrus ${ }^{1}$, \\ Jia Xian Law ${ }^{1} \mathbb{D}$, Muhammad Dain Yazid ${ }^{1}{ }^{\mathbb{D}}$, Kok-Yong Chin ${ }^{4} \mathbb{D}$, Sharen Aini Shamsuddin ${ }^{1}$ \\ and Yogeswaran Lokanathan $1, *$ (i)
}

check for updates

Citation: Chan, A.M.L.; Ng, A.M.H.; Mohd Yunus, M.H.; Idrus, R.B.H.; Law, J.X.; Yazid, M.D.; Chin, K.-Y.; Shamsuddin, S.A.; Lokanathan, Y. Recent Developments in Rodent Models of High-Fructose Diet-Induced Metabolic Syndrome: A Systematic Review. Nutrients 2021, 13, 2497. https://doi.org/10.3390/ nu13082497

\section{Academic Editors: Christina}

Chrysohoou and Konstantinos Tsioufis

Received: 10 July 2021

Accepted: 18 July 2021

Published: 22 July 2021

Publisher's Note: MDPI stays neutral with regard to jurisdictional claims in published maps and institutional affiliations.

Copyright: (c) 2021 by the authors. Licensee MDPI, Basel, Switzerland. This article is an open access article distributed under the terms and conditions of the Creative Commons Attribution (CC BY) license (https:// creativecommons.org/licenses/by/ $4.0 /)$.
1 Centre for Tissue Engineering and Regenerative Medicine, Faculty of Medicine, Universiti Kebangsaan Malaysia Medical Centre, Jalan Yaacob Latif, Kuala Lumpur 56000, Malaysia; alvinchanmanlung@outlook.com (A.M.L.C.); angela@ppukm.ukm.edu.my (A.M.H.N.); ruszymah@ppukm.ukm.edu.my (R.B.H.I.); lawjx@ppukm.ukm.edu.my (J.X.L.); dain@ukm.edu.my (M.D.Y.); s.sharenaini@ppukm.ukm.edu.my (S.A.S.)

2 Ming Medical Sdn. Bhd., D3-3 (2nd Floor), Block D3 Dana 1 Commercial Centre, Jalan PJU 1A/22, Petaling Jaya 47101, Malaysia

3 Department of Physiology, Faculty of Medicine, Universiti Kebangsaan Malaysia Medical Centre, Jalan Yaacob Latif, Cheras, Kuala Lumpur 56000, Malaysia; mohdheikalyunus@yahoo.com

4 Department of Pharmacology, Faculty of Medicine, Universiti Kebangsaan Malaysia Medical Centre, Jalan Yaacob Latif, Cheras, Kuala Lumpur 56000, Malaysia; chinkokyong@ppukm.ukm.edu.my

* Correspondence: lyoges@ppukm.ukm.edu.my; Tel.: +60-3-9145-7704

Abstract: Metabolic syndrome (MetS) is the physiological clustering of hypertension, hyperglycemia, hyperinsulinemia, dyslipidemia, and insulin resistance. The MetS-related chronic illnesses encompass obesity, the cardiovascular system, renal operation, hepatic function, oncology, and mortality. To perform pre-clinical research, it is imperative that these symptoms be successfully induced and optimized in lower taxonomy. Therefore, novel and future applications for a disease model, if proven valid, can be extrapolated to humans. MetS model establishment is evaluated based on the significance of selected test parameters, paradigm shifts from new discoveries, and the accessibility of the latest technology or advanced methodologies. Ultimately, the outcome of animal studies should be advantageous for human clinical trials and solidify their position in advanced medicine for clinicians to treat and adapt to serious or specific medical situations. Rodents (Rattus norvegicus and Mus musculus) have been ideal models for mammalian studies since the 18th century and have been mapped extensively. This review compiles and compares studies published in the past five years between the multitude of rodent comparative models. The response factors, niche parameters, and replicability of diet protocols are also compiled and analyzed to offer insight into MetS-related disease-specific modelling.

Keywords: high fructose; metabolic syndrome; syndrome X; metabolic syndrome X; rodent; rat; mice; mouse

\section{Introduction}

\subsection{Metabolic Syndrome}

The prevalence of obesity, diabetes, and cardiovascular diseases in modern society has been a global problem since the past century, and is still growing. Although "metabolic syndrome" (MetS) was coined in the early 1940s, the topic only became known after the works of Vague et al. and Haller and Hanfield, who successfully correlated the prevalence of obesity to diabetes and the hallmarks of MetS [1,2]. On 1 April 2020, the World Health Organization (WHO) reported that the prevalence of obesity had tripled over the four decades between 1975 and 2016 [3]. It was heavily implied that the low-to-mid-income countries are greatly affected by this and have since shown more linked deaths than underweight issues. Up to 1.9 billion adults ( $25 \%$ of the global population) have overweight 
problems. Higher and no variations between males (39\%) and females (40\%) have been reported. In the same year, the International Diabetes Federation (IDF) also reported that 223 million adults aged 20-79 years have diabetes [4]. This projection is expected to peak at 700 million by 2044 . Both organizations have also confirmed that the prevalence of obesity and diabetes has increased in children or adolescents, which is a major concern.

MetS is the phenomenon of accumulated symptoms that complementarily and progressively deteriorate the person's wellbeing. The contributing factors may vary between each person's exposure (environmental) or susceptibility because of hereditary (genetics) traits. Among the MetS contributors are unhealthy habits, sedentary lifestyle, poor diet choices, family history, socioeconomic status, and education [5]. Habits such as excessive alcohol consumption can cause hepatic dysfunction from the constant liver output of detoxifying metabolites. Smoking and narcotics are also common habits that deteriorate pulmonary and cardiac functions. A sedentary lifestyle is the privilege of access to advanced technology and entertainment that involves less physical stress and engagement. This drives the manifestation of obesity and the lack of mitochondrial stimulus for efficient energy production. By far, poor diet choices are the greatest contributor to MetS of the 21st century [3]. Previous correlations of the socioeconomic relationship of a person's income to obesity have shifted greatly, as recent findings show larger statistics in low-to-mid-income populations. The ease of accessibility to cheaper and hypercaloric diets driven by global franchises, as well as the incorporation of high salt, fat, and carbohydrate into traditional or commonly accessed foods, has seen to the growth of obesity. The traits of these nutritional imbalances can be defined as the Western diet phenomenon [6]. Conversely, the higher-income population has opted for a healthier and more organic lifestyle, which is not viable for the low-to-mid-income populations. Putting aside hereditary diseases such as type 1 diabetes, the role of genetics is less impactful, as it can be simplified as the person's susceptibility or tolerance of biochemical alterations. It is difficult to quantify the tolerance level, as it varies even among siblings, but can be controlled when a balanced diet and lifestyle are provided [7]. Lastly, education is better interpreted as self-awareness of healthy choices, habits, and the other factors as listed above. It is ultimately the person's discipline and restrained use of enriched resources to mitigate the effects of MetS.

The characteristics of MetS are high body mass index (BMI), hyperglycemia, hypertension, dyslipidemia, and insulin resistance. A joint interim statement from the IDF Task Force on Epidemiology and Prevention; National Heart, Lung, and Blood Institute; American Heart Association; World Heart Federation; International Atherosclerosis Society; and the International Association for the Study of Obesity states that a diagnosis of MetS is accepted if at least three of the five characteristics above are present [8]. A set of these conditions can lead to the development of major metabolic diseases such as cardiovascular diseases, type 2 diabetes, non-alcoholic fatty liver disease (NAFLD), osteoporosis, cancer, and death. Hence, the importance of studying MetS is to serve as a preliminary measure before the development of chronic disease. The present review was aimed at performing a qualitative analysis on the recent development of MetS in rodent models. It is intended to highlight the criteria for the successful establishment of an animal model of MetS. It is also aimed at compiling and comparing the test parameters for MetS and related diseases. Rodent models have been used extensively since the 18th century until today as the most preferred model for animal studies. Rodents are considerably easier to manage compared to larger mammalian families such as leporids, swine, equines, or primates. The selection of rodents is also driven by the ease of sourcing the animals; their lifespan, which is suited to the average study duration; and the complete mapping of their genetics and pathophysiological characteristics.

\subsection{Fructose as a Dietary Choice}

The present review focuses on diet-induced obesity (DIO) for simulating the development of obesity as a result of poor diet and lifestyle choices. One hallmark of DIO is the exaggerated incorporation of sugar, fat, and salt in the diet. References thereof 
are known as Western diets, introduced from regions that actively encourage chemical additives in processed foods, fast food franchises, and carbonated sugary beverages [9]. For example, the incorporation of fructose in beverages and processed food has been noticeably increasing. This popularization stems from fructose yielding a sweeter flavor, and it is most often added supplementary to generic sugar, i.e., sucrose, which is itself a disaccharide compound of glucose and fructose [10-12]. Fructose is one of three common sugars (monosaccharides) but is not directly processed in most metabolic processes such as energy (ATP) generation. Instead, fructose aids liver synthesis of glycogen molecules through a series of steps that overlaps with gluconeogenesis. In the context of MetS, fructose enhances the synthesis of triglycerides (TGL) from glycerol and fatty acid (FA) formation. Subsequently, TGL is stored as fat until a depletion of deposited glucose triggers a negative feedback. However, as glucose scarcity is less probable, the metabolized fructose is deemed in excess and is stored principally as fat. Evidence has been presented indicating that fructose bears similarity to narcotics, enabling unhealthy compulsion and downstream hyperphagia $[9,13,14]$. In this context, high fructose consumption is associated with MetS prevalence.

\subsection{Fructolysis}

The machination of fructolysis begins through the diffusion of fructose by the transporter GLUT5 in a concentration-dependent manner. Aided by GLUT2, it crosses the intestinal lumen walls to exit to the bloodstream. As blood courses throughout the body, the fructose is bound by the GLUT2 transporters of the liver $[11,15,16]$. Therein, fructokinase catalyzes fructose into fructose-1-phosphate (F1P). In turn, F1P is cleaved by aldolase B into dihydroxyacetone phosphate (DHAP) and glyceraldehyde (Figure 1). Subsequently, DHAP is converted into glyceraldehyde-3-phosphate (G3P), pyruvate, acetyl-CoA, and FA molecules. Meanwhile, glyceraldehyde is first converted into glycerol, then glycerol-3phosphate (Gro3P). Finally, TGL is formed following Gro3P and FA esterification. TGL and FA are both released into the bloodstream, contributing to dyslipidemia, which is defined as abnormal lipid levels in the blood [17,18].

Between the course of pyruvate conversion to acetyl-CoA, citrate, $\mathrm{CO}_{2}$, and ATP molecules are released. Citrate and ATP act on the phosphofructokinase (PFK) enzyme (of the glycolysis mechanism), responsible for phosphorylating fructose-6-phosphate (F6P) into fructose-1,6-bisphosphate (FBP). ATP activates PFK to stimulate functions while citrate inhibits it via the PFK affinity for ATP binding. The pathway of fructolysis is able to circumvent this crucial regulatory process, which is a devastating concern since there are absent or minimal downstream homeostatic mechanisms that are able to reverse or attenuate said impact $[9-11,14,15]$. Thus, its effects widely impact many physiological systems, with potentiating acute to chronic levels of damage.

As mentioned above, the primary product of fructolysis is TGL. Among the eventual outcomes of metabolic disorders, the damage would likely stem from the organs responsible for blood circulation and filtration. Hence, the liver, kidney, pancreas, and vascular system are the earliest victims of chronic fructose ingestion [15,19]. Excess adipogenesis and lipogenesis will precipitate increased adipocytokines, such as leptin. Although leptin balances energy homeostasis through hunger, overproduction will accumulate as leptin resistance. Consequently, this leads to hyperphagia, obesity, and increased percentage of fat mass [19]. Adipocytokines also consist of inflammatory cytokines. The commonly referred MetS cytokines are tumor necrosis factor alpha (TNF- $\alpha$ ), interleukin-6 (IL-6), and reactive oxygen species (ROS) or antioxidants. The fluctuating production of these metabolites can lead to inflammation, insulin resistance, and hypertension [20-23]. Individually, each of these adipo-secretomes exerts mild effects, but may collectively result in one or more systemic complications. 


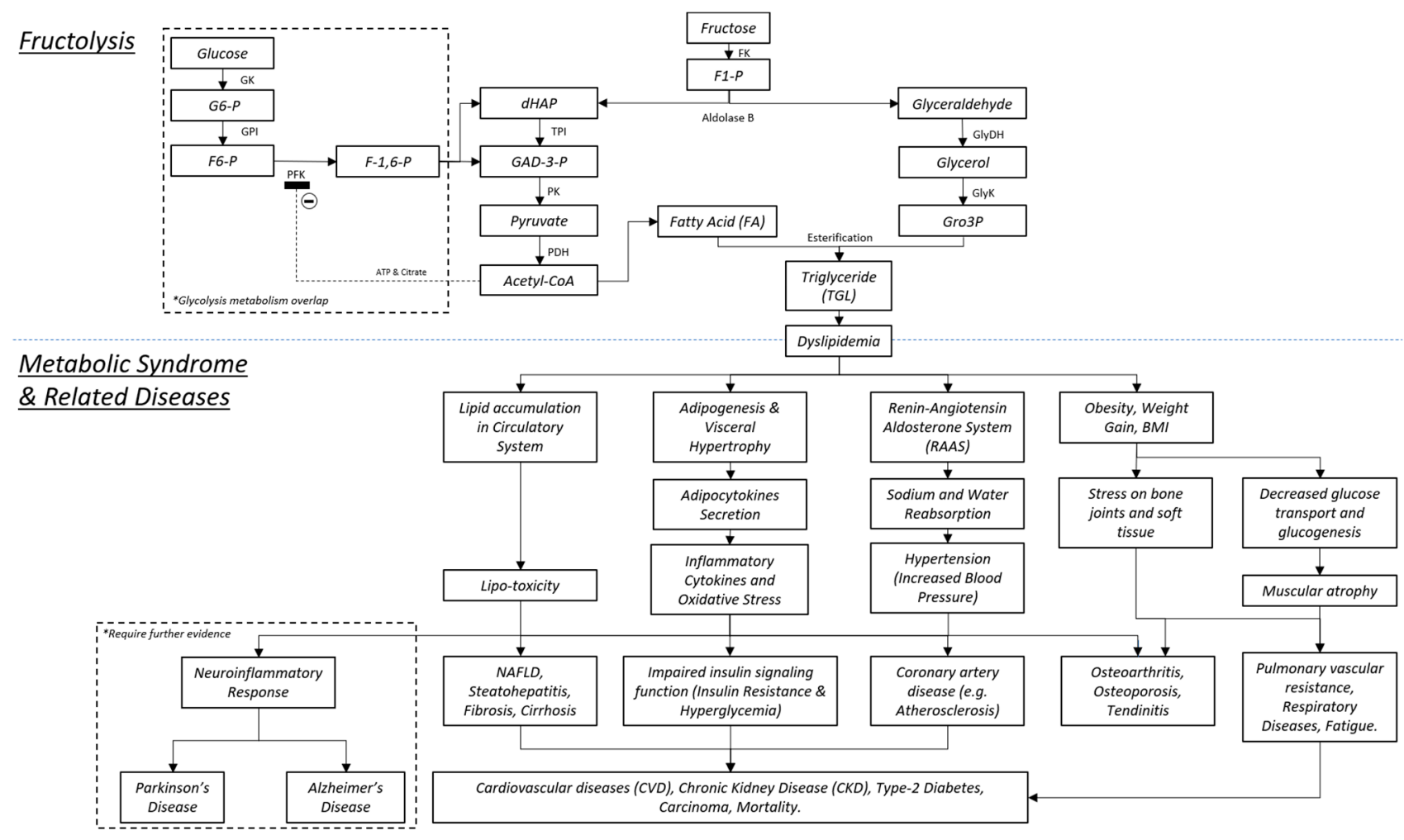

Figure 1. Fructose enzymatic process and physiological impacts to achieve MetS and related diseases. Abbreviations: GK, glucosekinase; G6-P, glucose-6-phosphate; GPI, phosphoglucose isomerase; PFK, phosphofructokinase-1; F-1,6-P, fructose1,6-phosphate; dHAP, dihydroxyacetone phosphate; TPi, triophosphate isomerase; GAD-3-P, glyceraldehyde-3-phosphate; PK, phosphofructokinase; PDH, pyruvate dehydrogenase; FK, fructokinase; F1-P, fructose-1-phosphate; GlyDH, glycerol dehydrogenase, GlyK, glycerate kinase.

\subsection{The Bone and MetS}

In Figure 1, the focus of MetS is centered on insulin resistance and/or inflammatory cytokines. Therefore, the categories of liver, cardiovascular, renal, and oncological diseases are the highlights. The inclusion of bone diseases is an extremely intriguing premise because of the condition that ageing and sex-favoring disease are minimized. Osteoporosis has significantly greater incidence in women than in men, more so with ageing co-factored simultaneously. Contradictory to current beliefs, men and young adults of both sexes have osteoporosis risk derived from severe MetS [24,25]. Although MetS has been reported with non-significant values, it has been noted that increased weight gain, adiposity, and oxidative stress are nevertheless potential contributors [26-29]. As the topic is highly appraised, the notion of bone-related diseases experiencing a paradigm shift by MetS is a major concern for other factor-limited diseases. That the expanding efforts in MetS will provide further clarity is of interest.

\subsection{The Brain and MetS}

There have been equal postulations of MetS in relation to cognitive decline and unhealthy pressure on neurological diseases. Globally, two of the most common agerelated metabolic deteriorations of the brain are Alzheimer disease and Parkinson disease. Several authors have explored the impact of disproportionate inflammatory secretions and oxidative reduction on accelerating or aggravating the encephalic system [30,31]. Insulin resistance and irresponsive receptors in the brain significantly decrease blood flow, leading to chronic oxidative stress and damaged cognitive roles [30,31]. However, this premise is challenged due to insufficient evidence and significant values [32]. Based on these studies, minimal to absent efforts in genomic and proteomic studies are apparent, although this may be an issue of accessibility to advanced methodology or technology during the past 
decades. Hence, this is another research topic to be rediscovered. If there is much evidence, novel studies could pave the foundation in neurology and gerontology towards novel therapeutics for neurodegenerative and mental disorders.

\section{Materials and Methods}

The methodology for this systematic review was approved and registered under the guidelines of PROSPERO (International prospective register of systematic review, PROSPERO ID: CRD42021238988).

\subsection{Keyword Selection}

To identify the keyword validity and synonyms, "Metabolic Syndrome" and "High Fructose" were searched through medical subject headings (MeSH), a PubMed vocabulary thesaurus. The keyword search results served as standard search terminologies for articles.

\subsection{Database Selection and Result Filtering}

The Scopus, PubMed, and Web Of Science (WOS) databases were selected from available access provided by the National University of Malaysia Faculty of Medicine. The keywords were searched and the databases were filtered specifically for "research articles" or "journal articles" published in 2016-2020 (5 years). The bibliographies were downloaded from the respective databases and labelled appropriately (e.g., PUBMED_12_11_2020 109 results).

\subsection{Inclusion of Titles, Abstracts, and Keywords}

The bibliographies were uploaded to citation software (Mendeley). Files were downloaded separately, compiled in a different folder, and duplicates were combined. Duplicates were removed automatically but were also removed manually for assurance. The titles and abstracts were initially screened according to the inclusion and exclusion criteria. The inclusion criteria were: "high fructose" and "metabolic syndrome" and $\geq 3$ MetS symptoms: (i) Increased weight or abdominal circumference, (ii) dyslipidemia, (iii) hypertension, (iv) decreased high-density lipoprotein (HDL) levels, and (v) hyperglycemia. The exclusion criteria were: (i) long-term study or aging effect, (ii) generational study, (iii) absent metabolic disorders or parameters, (iv) non-fructose diet, and (v) high fructose in combination with other diet.

\subsection{Inclusion of Articles Based on Methods and Results}

A second screening was performed by evaluating the method for fructose-only MetS induction. For example, the combination of high-fat, high-salt, and high-carbohydrate diets with fructose was excluded. Next, the results of individual studies must also have presented a valid comparison of fructose diets to control diets before any treatment. All excluded and included studies were retained and numbered for totals (Figure 2).

Figure 2 shows that the initial search yielded 109 articles from PubMed, 361 articles from Scopus, and 256 articles from WOS. In total, the search yielded 726 articles when combined. Duplicate auto-removal was performed upon combination, followed by manual duplicate removal. The total pooled database numbered 597 individual articles, and 129 duplicates were removed. Two reviewers individually filtered the articles following the agreed parameters. Although both reviewers had 18 articles each, four articles were not identical. Post-discussion, a total of 18 articles were agreed for further review. The articles reviewed are presented as below (Table 1), in chronological years (earliest to recent). 
Table 1. Main factors attributed to MetS establishment in rodent models.

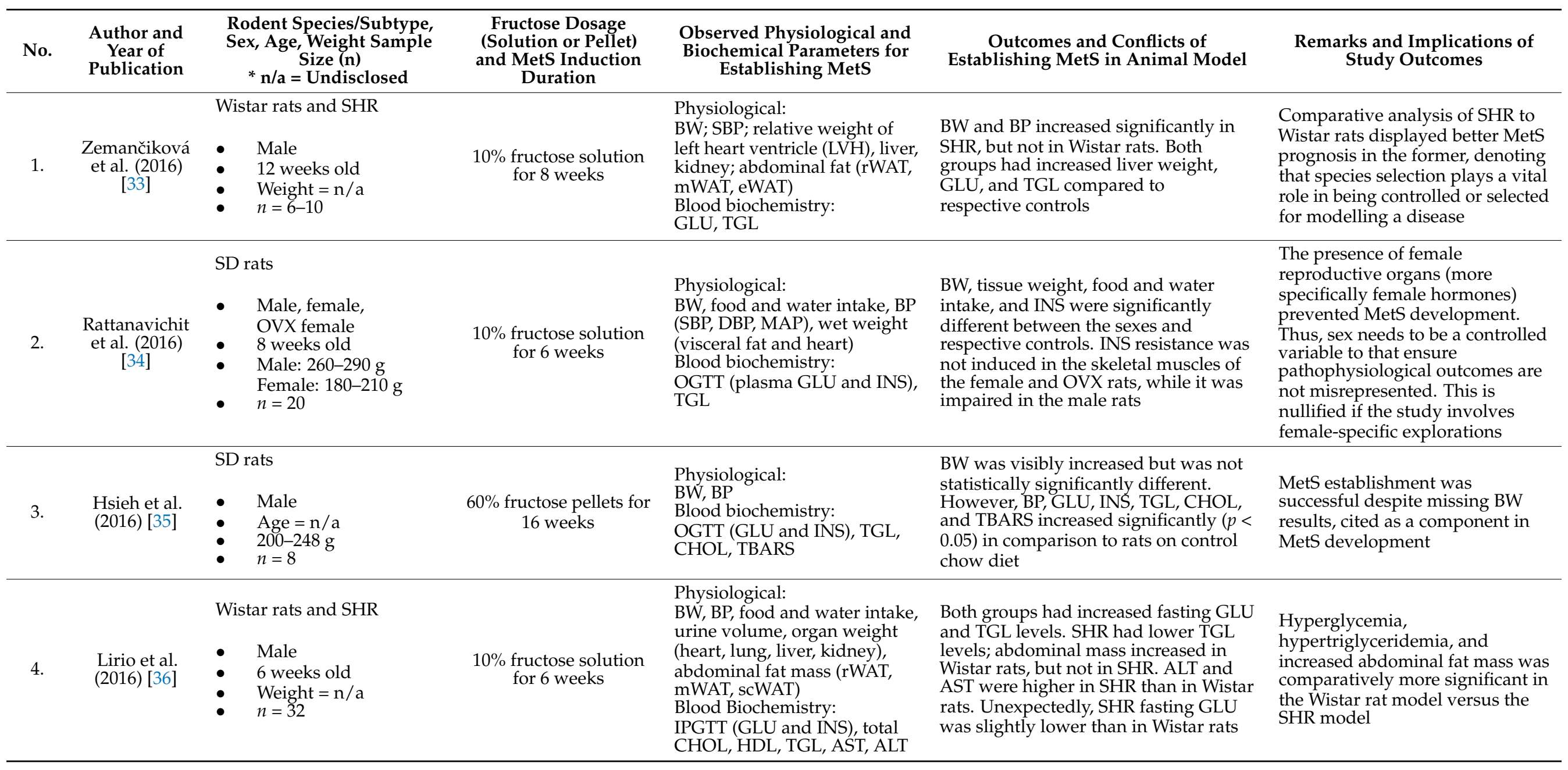


Table 1. Cont.

\begin{tabular}{|c|c|c|c|c|c|c|}
\hline No. & $\begin{array}{l}\text { Author and } \\
\text { Year of } \\
\text { Publication }\end{array}$ & $\begin{array}{c}\text { Rodent Species/Subtype, } \\
\text { Sex, Age, Weight Sample } \\
\text { Size (n) } \\
* \text { n/a = Undisclosed }\end{array}$ & $\begin{array}{c}\text { Fructose Dosage } \\
\text { (Solution or Pellet) } \\
\text { and MetS Induction } \\
\text { Duration }\end{array}$ & $\begin{array}{l}\text { Observed Physiological and } \\
\text { Biochemical Parameters for } \\
\text { Establishing MetS }\end{array}$ & $\begin{array}{l}\text { Outcomes and Conflicts of } \\
\text { Establishing MetS in Animal Model }\end{array}$ & $\begin{array}{l}\text { Remarks and Implications of } \\
\text { Study Outcomes }\end{array}$ \\
\hline 5. & $\begin{array}{l}\text { Bargut et al. } \\
\text { (2017) [20] }\end{array}$ & $\begin{array}{l}\text { CB57BL } / 6 \text { mice } \\
\text { - } \quad \text { Male } \\
\text { - } \quad 3 \text { months old } \\
\text { - } \quad \text { Weight }=\mathrm{n} / \mathrm{a} \\
\quad n=48\end{array}$ & $\begin{array}{l}46.43 \% \text { fructose pellets } \\
\text { for } 3 \text { weeks }\end{array}$ & $\begin{array}{l}\text { Physiological: } \\
\text { BW, BP, food and water } \\
\text { intake, eWAT } \\
\text { Blood biochemistry: } \\
\text { OGTT, TGL, CHOL, INS, } \\
\text { HOMA-IR, Adipo, LEP }\end{array}$ & $\begin{array}{l}\text { BP, TGL, CHOL, INS, HOMA-IR, and } \\
\text { LEP were increased significantly; Adipo } \\
\text { was decreased in the Hfru group } \\
\text { compared to control group }\end{array}$ & $\begin{array}{l}\text { Although physical aspects such as } \\
\text { BW and fat mass were not } \\
\text { statistically significantly } \\
\text { differentiated, Hfru } \\
\text { induced hypertension, } \\
\text { hypertriglyceridemia, } \\
\text { hypercholesterolemia, and } \\
\text { INS resistance }\end{array}$ \\
\hline 6. & $\begin{array}{l}\text { Bratoeva et al. } \\
\quad(2018) \text { [37] }\end{array}$ & $\begin{array}{l}\text { Wistar rats } \\
\quad \quad \text { Male } \\
: \quad \text { Age }=\mathrm{n} / \mathrm{a} \\
\quad 140-180 \mathrm{~g} \\
\quad n=14\end{array}$ & $\begin{array}{l}35 \% \text { fructose solution } \\
\text { for } 16 \text { weeks }\end{array}$ & $\begin{array}{l}\text { Physiological: } \\
\text { BW, organ weight } \\
\text { Blood biochemistry: } \\
\text { GLU, TGL, TP, Urea, UA, } \\
\text { CREA, electrolyte (Na and K), } \\
\text { GSH, MDA, CRP }\end{array}$ & $\begin{array}{l}\text { BW, kidney weight, GLUC, TGL, MDA, } \\
\text { GSH, UA, and CRP } \\
\text { increased significantly }\end{array}$ & $\begin{array}{l}\text { BW increased, hyperglycemia, } \\
\text { hypertriglyceridemia, } \\
\text { hyperuricemia, and oxidative } \\
\text { stress were achieved. The } \\
\text { addition of HOMA-IR could be } \\
\text { considered, as INS resistance is a } \\
\text { notable hallmark of MetS }\end{array}$ \\
\hline 7. & $\begin{array}{l}\text { Ramos et al. } \\
\text { (2017) [38] }\end{array}$ & $\begin{array}{l}\text { Wistar rats } \\
\text { - } \quad \text { Male } \\
\text { - } \quad 30 \text { days old } \\
\text { - } \quad 100-148 \mathrm{~g} \\
\quad n=20\end{array}$ & $\begin{array}{l}20 \% \text { fructose solution } \\
\text { for } 30,60 \text {, and } 90 \text { days }\end{array}$ & $\begin{array}{l}\text { Physiological: } \\
\text { BW, food and water intake, } \\
\text { energy intake, feed efficiency, } \\
\text { fat mass (eWAT, mWAT, } \\
\text { rWAT), total adipose mass } \\
\text { Blood biochemistry: } \\
\text { GLU, TGL }\end{array}$ & $\begin{array}{l}\text { Increased BW, fat mass, TGL levels. } \\
\text { Total fat mass and eWAT and rWAT } \\
\text { deposits were significantly greater in } \\
\text { the fructose-fed groups } \\
\text { Food and water intake were lower in } \\
\text { the Hfru group upon the } \\
\text { induction period }\end{array}$ & $\begin{array}{l}\text { Overall, Hfru induced increased } \\
\text { BW and adipogenesis, and } \\
\text { hypertriglyceridemia } \\
\text { Only hyperglycemia was } \\
\text { not achieved }\end{array}$ \\
\hline 8. & $\begin{array}{l}\text { Abdelrahman } \\
\text { et al. (2018) } \\
\text { [21] }\end{array}$ & $\begin{array}{l}\text { Wistar rats } \\
\quad \quad \text { Male } \\
: \quad \text { Age }=\mathrm{n} / \mathrm{a} \\
\quad 180-290 \mathrm{~g} \\
\quad n=24\end{array}$ & $\begin{array}{l}60 \% \text { fructose pellets for } \\
5 \text { weeks }\end{array}$ & $\begin{array}{l}\text { Physiological: } \\
\text { BW, BW change, water intake, } \\
\text { urine output, BP, heart rate, } \\
\text { weight of abdominal aorta } \\
\text { Blood biochemistry: } \\
\text { GLU, INS, HOMA-IR, TGL, } \\
\text { CHOL, HDL, LDL, UA }\end{array}$ & $\begin{array}{l}\text { Water intake and output decreased } \\
\text { significantly. Fructose diet incited } \\
\text { hypertension, hyperinsulinemia, } \\
\text { hypertriglyceridemia, and } \\
\text { hypercholesterolemia }\end{array}$ & $\begin{array}{l}\text { MetS was successfully induced in } \\
\text { the model, with the exception of } \\
\text { BW, which was maintained } \\
\text { throughout the } \\
\text { experimental phase }\end{array}$ \\
\hline
\end{tabular}


Table 1. Cont.

\begin{tabular}{|c|c|c|c|c|c|c|}
\hline No. & $\begin{array}{l}\text { Author and } \\
\text { Year of } \\
\text { Publication }\end{array}$ & $\begin{array}{c}\text { Rodent Species/Subtype, } \\
\text { Sex, Age, Weight Sample } \\
\text { Size (n) } \\
* \text { n/a = Undisclosed }\end{array}$ & $\begin{array}{c}\text { Fructose Dosage } \\
\text { (Solution or Pellet) } \\
\text { and MetS Induction } \\
\text { Duration }\end{array}$ & $\begin{array}{l}\text { Observed Physiological and } \\
\text { Biochemical Parameters for } \\
\text { Establishing MetS }\end{array}$ & $\begin{array}{l}\text { Outcomes and Conflicts of } \\
\text { Establishing MetS in Animal Model }\end{array}$ & $\begin{array}{l}\text { Remarks and Implications of } \\
\text { Study Outcomes }\end{array}$ \\
\hline 9. & $\begin{array}{l}\text { Ng et al. } \\
(2018)[39]\end{array}$ & $\begin{array}{l}\text { SD rats } \\
\quad \text { Male } \\
: \quad \text { Age }=\mathrm{n} / \mathrm{a} \\
\quad 180-200 \mathrm{~g} \\
\quad n=24\end{array}$ & $\begin{array}{l}60 \% \text { fructose pellets for } \\
3 \text { and } 5 \text { months (FR- } 3 \\
\text { and FR-5, respectively) }\end{array}$ & $\begin{array}{l}\text { Physiological: } \\
\text { BW, tissue weight, BP, urine } \\
\text { output } \\
\text { Blood biochemistry: } \\
\text { Plasma GLU, UA, CHOL, } \\
\text { TGL, CREA, electrolytes (Na } \\
\text { and K) }\end{array}$ & $\begin{array}{l}\text { BW and UA were significantly higher in } \\
\text { both FR- } 3 \text { and FR- } 5 \text { as compared to the } \\
\text { control only. BP, UA, TGL, and CHOL } \\
\text { were higher in FR- } 5 \text { compared to FR-3 } \\
\text { and the control. Conversely, fasting } \\
\text { GLU was higher at } 3 \text { months than } \\
5 \text { months }\end{array}$ & $\begin{array}{l}\text { Overall, fructose feeding enabled } \\
\text { increased BP and proteinuria, but } \\
\text { only hypercholesterolemia was } \\
\text { significant by } 5 \text { months. This } \\
\text { study highlights the importance } \\
\text { of the duration in inducing MetS } \\
\text { to be acceptably significant for } \\
\text { research purposes. In comparison } \\
\text { to other studies, this duration is } \\
\text { deemed overtly lengthy, possibly } \\
\text { allowing age to be a confounding } \\
\text { factor in MetS development }\end{array}$ \\
\hline 10 & $\begin{array}{l}\text { Chen et al. } \\
(2018)[40]\end{array}$ & $\begin{array}{l}\text { SD rats } \\
\qquad \quad \text { Male } \\
\bullet \quad 10 \text { weeks old } \\
\bullet \quad 200-248 \mathrm{~g} \\
\bullet \quad n=24\end{array}$ & $\begin{array}{l}60 \% \text { fructose pellet for } \\
9 \text { weeks }\end{array}$ & $\begin{array}{l}\text { Physiological: } \\
\text { BW, BP } \\
\text { Blood biochemistry: } \\
\text { Plasma GLU, INS, } \\
\text { CHOL, TGL }\end{array}$ & $\begin{array}{l}\text { Fructose-fed rats exhibited increased } \\
\text { BW, GLUC, and BP }(p<0.01) \text {. Similarly, } \\
\text { INS, CHOL, and TGL levels increased } \\
\text { significantly }(p<0.001)\end{array}$ & $\begin{array}{l}\text { Long-term 9-week feeding } \\
\text { induced significant levels of } \\
\text { obesity, hyperglycemia, } \\
\text { hyperinsulinemia, hypertension, } \\
\text { and hyperlipidemia }\end{array}$ \\
\hline 11. & $\begin{array}{c}\text { Gambaro } \\
\text { et al. (2018) } \\
{[22]}\end{array}$ & 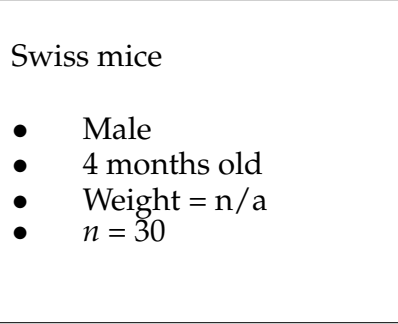 & $\begin{array}{l}20 \% \text { fructose solution } \\
\text { for } 6 \text { and } 10 \text { weeks }\end{array}$ & $\begin{array}{l}\text { Physiological: } \\
\text { BW, food and water intake, BP, } \\
\text { adipose tissue weight (iWAT, } \\
\text { eWAT, rWAT), liver weight } \\
\text { Blood biochemistry: } \\
\text { GLU, OGTT, AUC, TGL, LEP, } \\
\text { ALT, AST }\end{array}$ & $\begin{array}{l}\text { During both durations, BW, AUC, TGL, } \\
\text { and LEP were significantly increased; } \\
\text { food intake decreased. Hfru plasma } \\
\text { LEP and only ALT were significantly } \\
\text { higher at } 10 \text { weeks than } 6 \text { weeks }\end{array}$ & $\begin{array}{l}\text { Food intake was superseded } \\
\text { despite being decreased, as Hfru } \\
\text { would have larger feed efficiency } \\
\text { due to a hypercaloric diet. The } \\
\text { results of induction were } \\
\text { substantially reliable by week } 10 \text {. } \\
\text { Hyperglycemia was not achieved } \\
\text { in either induction period when } \\
\text { compared to the control }\end{array}$ \\
\hline 12. & $\begin{array}{l}\text { Subramani } \\
\text { et al. (2019) } \\
\text { [41] }\end{array}$ & 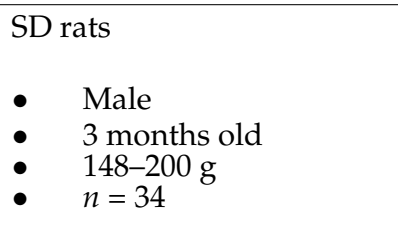 & $\begin{array}{l}66 \% \text { Hfru pellets for } \\
6 \text { weeks }\end{array}$ & $\begin{array}{l}\text { Physiological: } \\
\text { BW, body length, BMI, BP } \\
\text { Blood biochemistry: } \\
\text { Fasting GLU, serum lipid } \\
\text { profile (CHOL, TGL, } \\
\text { HDL, LDL) }\end{array}$ & $\begin{array}{l}\text { After } 6 \text { weeks, Hfru feeding increased } \\
\text { BMI, GLU, MAP, TGL, CHOL, and LDL } \\
\text { compared to the control. Expectedly, } \\
\text { HDL decreased compared to the control }\end{array}$ & $\begin{array}{l}\text { Hfru feeding successfully induced } \\
\text { weight gain, hypertension, } \\
\text { hyperglycemia, and } \\
\text { hyperlipidemia }\end{array}$ \\
\hline
\end{tabular}


Table 1. Cont.

\begin{tabular}{|c|c|c|c|c|c|c|}
\hline No. & $\begin{array}{l}\text { Author and } \\
\text { Year of } \\
\text { Publication }\end{array}$ & $\begin{array}{c}\text { Rodent Species/Subtype, } \\
\text { Sex, Age, Weight Sample } \\
\text { Size (n) } \\
* \text { n/a = Undisclosed }\end{array}$ & $\begin{array}{c}\text { Fructose Dosage } \\
\text { (Solution or Pellet) } \\
\text { and MetS Induction } \\
\text { Duration }\end{array}$ & $\begin{array}{l}\text { Observed Physiological and } \\
\text { Biochemical Parameters for } \\
\text { Establishing MetS }\end{array}$ & $\begin{array}{l}\text { Outcomes and Conflicts of } \\
\text { Establishing MetS in Animal Model }\end{array}$ & $\begin{array}{l}\text { Remarks and Implications of } \\
\text { Study Outcomes }\end{array}$ \\
\hline 13. & $\begin{array}{l}\text { Fakhoury- } \\
\text { Sayegh et al. } \\
\text { (2019) [23] }\end{array}$ & $\begin{array}{l}\text { Wistar rats } \\
\text { - } \quad \text { Male } \\
\text { - } \quad 6 \text { weeks old } \\
\text { - } \quad 148-200 \mathrm{~g} \\
\quad n=40\end{array}$ & $\begin{array}{c}10 \%, 20 \% \text {, and } 30 \% \\
\text { fructose pellets for } \\
16 \text { weeks }\end{array}$ & $\begin{array}{l}\text { Physiological: } \\
\text { BW, food intake, energy } \\
\text { intake, organ weight (liver, } \\
\text { kidney, pancreas, eWAT) } \\
\text { Blood biochemistry: } \\
\text { Fasting GLU, INS, TGL, } \\
\text { ALT, AST }\end{array}$ & $\begin{array}{l}\text { After } 16 \text { weeks, serum GLU increased } \\
\text { in Hfru groups compared to the control. } \\
\text { AST decreased in } 20 \% \text { and } 30 \% \text { Hfru } \\
\text { groups only but INS levels were } \\
\text { increased in the } 10 \% \text { Hfru group. ALT } \\
\text { only increased in the control and } 10 \% \\
\text { Hfru groups }\end{array}$ & $\begin{array}{l}\text { This study utilized the lowest } \\
\text { concentrations of fructose in pellet } \\
\text { diet paired with the longest } \\
\text { induction duration to establish the } \\
\text { minimum threshold for NAFLD, a } \\
\text { MetS-related disease. For a } \\
\text { long-term study, this may perhaps } \\
\text { be applicable, but in most studies, } \\
\text { it is desirable to achieve effects in } \\
\text { an optimal duration }\end{array}$ \\
\hline 14. & $\begin{array}{l}\text { Zhang et al. } \\
(2020)[42]\end{array}$ & $\begin{array}{l}\text { C57BL/6J, DBA/2 J (DBA), } \\
\text { FVB/NJ (FVB) mice } \\
\text { - } \quad \text { Male } \\
\bullet \quad 8 \text { weeks old } \\
\bullet \quad 20-25 \mathrm{~g} \\
-\quad n=8-12 \text { per } \\
\quad \text { strain Control }=8-10 \\
\quad \text { per strain }\end{array}$ & $\begin{array}{l}8 \% \text { fructose solution } \\
\text { for } 12 \text { weeks }\end{array}$ & $\begin{array}{l}\text { Physiological: } \\
\text { BW; food intake; caloric intake; } \\
\text { organ weight (hypothalamus } \\
\text { and liver); lean and fat mass of } \\
\text { rWAT, mWAT, and scWAT. } \\
\text { Blood biochemistry: } \\
\text { IPGTT, plasma INS, } \\
\text { GLU, lipids }\end{array}$ & $\begin{array}{l}\text { Only DBA mice gained significant BW, } \\
\text { fat mass, and fat mass percentage; } \\
\text { percentage of lean mass was decreased } \\
\text { (increased fat mass ratio); rWAT, mWAT } \\
\text { and scWAT weight was increase; GLU } \\
\text { homeostasis levels were impaired } \\
\text { compared to the C57BL/6J and FVB } \\
\text { mice. Plasma INS was significantly } \\
\text { elevated in DBA and FVB mice. All } \\
\text { groups had increased fructose/water } \\
\text { intake and decreased food intake but } \\
\text { maintained caloric intake }\end{array}$ & $\begin{array}{l}\text { This study elucidated the } \\
\text { comparison between } M \text {. musculus } \\
\text { strains; the final outcome was that } \\
\text { DBA mice demonstrated greater } \\
\text { susceptibility to chronic fructose } \\
\text { ingestion than a SHR model. }\end{array}$ \\
\hline 15. & $\begin{array}{l}\text { Mustafa et al. } \\
\text { (2020) [43] }\end{array}$ & 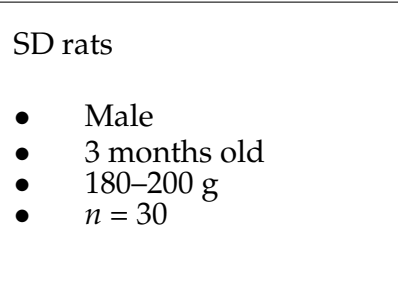 & $\begin{array}{l}20 \%(\mathrm{~F} 20 \%) \text { and } 40 \% \\
\text { fructose (F40\%) } \\
\text { solution for } 6 \text { weeks }\end{array}$ & $\begin{array}{l}\text { Physiological: } \\
\text { BW, body length, abdominal } \\
\text { circumference, BMI } \\
\text { Blood biochemistry: } \\
\text { GLU, INS, HOMA-IR, TP, Alb, } \\
\text { Gb, urea, CREA, ALT, AST, } \\
\text { BIL, lipid profile (TGL, VLDL, } \\
\text { HDL, LDL) }\end{array}$ & $\begin{array}{l}\text { BW, BW gained (\%), and BMI were } \\
\text { statistically significantly increased } \\
\text { compared to the control. Urea and } \\
\text { CREA were increased significantly in } \\
\text { the F40\% group only. AST, BIL, TGL, } \\
\text { and HDL were reduced; VLDL and } \\
\text { LDL were increased significantly } \\
\text { compared to the control }\end{array}$ & $\begin{array}{l}\text { The MetS diet-induced model was } \\
\text { successful through } 40 \% \text { fructose } \\
\text { solution for } 6 \text { weeks while } 20 \% \\
\text { fructose solution was insufficient. } \\
\text { The animal model demonstrated } \\
\text { insulin resistance, obesity, } \\
\text { dyslipidemia, metabolic } \\
\text { imbalance, and inflammation }\end{array}$ \\
\hline
\end{tabular}


Table 1. Cont.

\begin{tabular}{|c|c|c|c|c|c|c|}
\hline No. & $\begin{array}{l}\text { Author and } \\
\text { Year of } \\
\text { Publication }\end{array}$ & $\begin{array}{c}\text { Rodent Species/Subtype, } \\
\text { Sex, Age, Weight Sample } \\
\text { Size (n) } \\
* \text { n/a = Undisclosed }\end{array}$ & $\begin{array}{c}\text { Fructose Dosage } \\
\text { (Solution or Pellet) } \\
\text { and MetS Induction } \\
\text { Duration }\end{array}$ & $\begin{array}{l}\text { Observed Physiological and } \\
\text { Biochemical Parameters for } \\
\text { Establishing MetS }\end{array}$ & $\begin{array}{l}\text { Outcomes and Conflicts of } \\
\text { Establishing MetS in Animal Model }\end{array}$ & $\begin{array}{l}\text { Remarks and Implications of } \\
\text { Study Outcomes }\end{array}$ \\
\hline 16. & $\begin{array}{l}\text { Ferreira- } \\
\text { Santos et al. } \\
\text { (2020) [44] }\end{array}$ & $\begin{array}{l}\text { Wistar rats } \\
\text { - } \quad \text { Male } \\
\text { - } \quad 8 \text { weeks old } \\
\text { - } \quad n=25 \text { g } \\
\quad n=28\end{array}$ & $\begin{array}{l}20 \% \text { fructose solution } \\
\text { for } 12 \text { weeks }\end{array}$ & $\begin{array}{l}\text { Physiological: } \\
\text { BW, caloric intake, organ } \\
\text { weight (heart, liver, abdominal } \\
\text { fat, mesenteric, aorta artery), } \\
\text { tibia length, SBP } \\
\text { Blood biochemistry: } \\
\text { OGTT, fasting blood GLU, } \\
\text { INS, AUC, HOMA-IR, lipid } \\
\text { profile (TGL CHOL, HDL, } \\
\text { LDL), AST, ALT }\end{array}$ & $\begin{array}{l}\text { SBP, GLUC, AUC, INS, HOMA-IR, } \\
\text { abdominal fat mass, TGL, CHOL, ALT, } \\
\text { AST, and BMI were increased } \\
\text { significantly in the fructose group } \\
\text { compared to the control }\end{array}$ & $\begin{array}{l}\text { The Wistar model struggled to } \\
\text { gain physical weight although } \\
\text { increased organ weight, fat mass } \\
\text { accumulation, hyperglycemia, } \\
\text { dyslipidemia, and inflammation } \\
\text { were achieved }\end{array}$ \\
\hline 17. & $\begin{array}{l}\text { Kitagawa } \\
\text { et al. (2020) } \\
{[45]}\end{array}$ & $\begin{array}{l}\text { Wistar rats } \\
\text { - } \quad \text { Male } \\
\text { - } \quad 4 \text { weeks old } \\
\text { - } \quad \text { Weight = n/a } \\
\quad n=13\end{array}$ & $\begin{array}{l}60 \% \text { fructose pellets for } \\
2,4 \text {, and } 6 \text { weeks }\end{array}$ & $\begin{array}{l}\text { Physiological: } \\
\text { BW, organ weight (liver, } \\
\text { kidney, heart, biceps } \\
\text { femoris, eWAT) } \\
\text { Blood biochemistry: } \\
\text { HOMA-IR (GLU and INS), } \\
\text { TGL, CHOL, FFA }\end{array}$ & $\begin{array}{l}\text { HOMA-IR, TGL, CHOL, and FFA } \\
\text { increased during week } 2 \text { and continued } \\
\text { the increasing trajectory during week } 4 \\
\text { and further by } 6 \text { weeks of feeding }\end{array}$ & $\begin{array}{l}\text { Weight gain and hyperglycemia } \\
\text { were not achieved. However, IR, } \\
\text { hyperinsulinemia, and } \\
\text { dyslipidemia were achieved in } \\
\text { this animal model }\end{array}$ \\
\hline
\end{tabular}

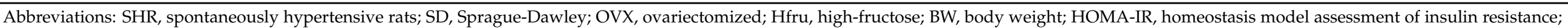

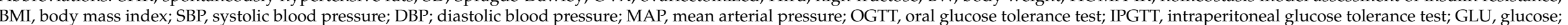

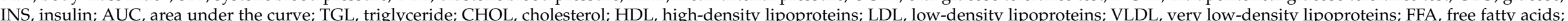

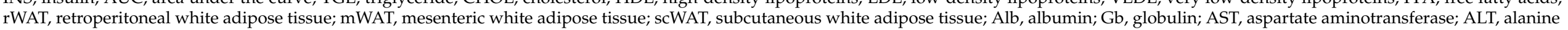

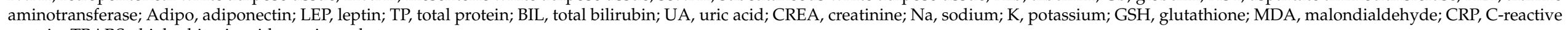
protein; TBARS, thiobarbituric acid-reactive substances. 


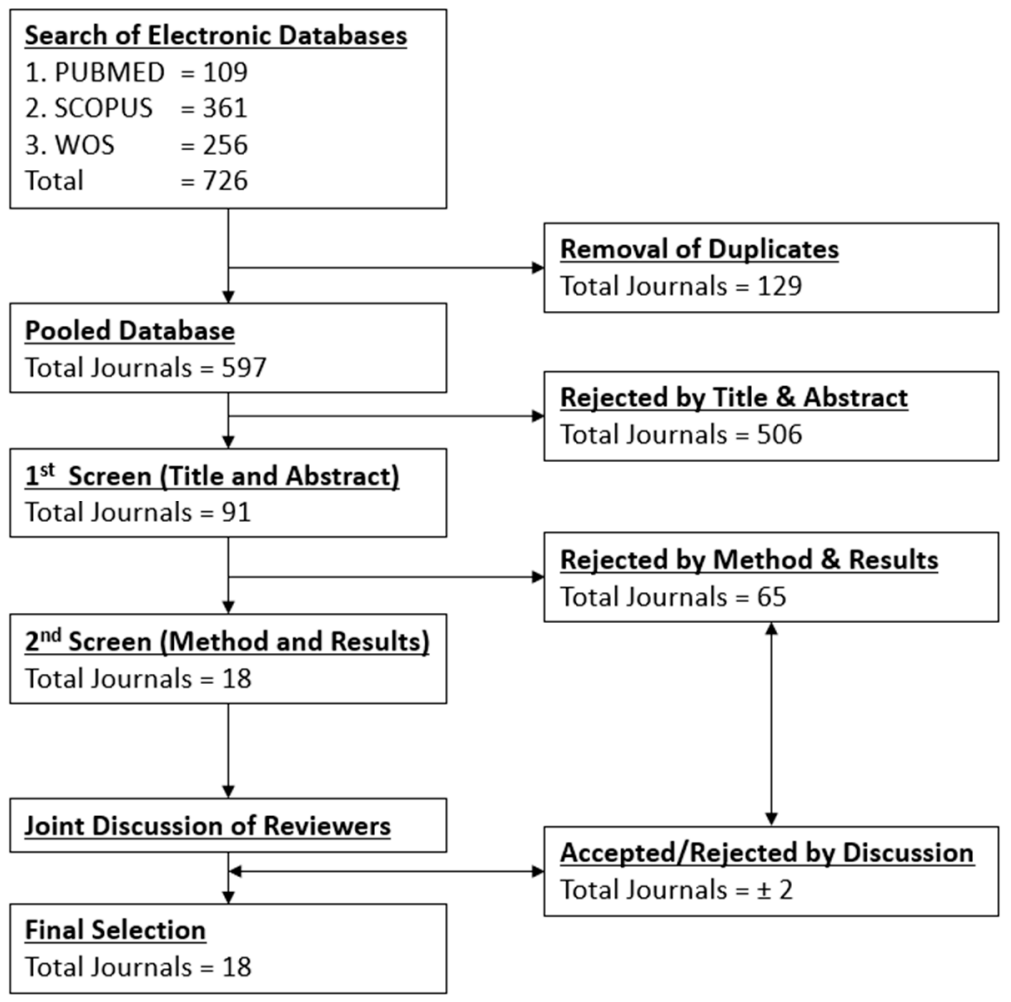

Figure 2. PRISMA flow diagram for process of article selection from pooled database of PUBMED, SCOPUS and WoS.

\section{Results and Discussion}

\subsection{Rodent Selection Criteria}

\subsubsection{Susceptibility of Specific Rodent Strains}

Rodent selection is an important factor in simulating MetS in an animal model. Species and sex variability should be controlled based on the requirements of the experiment. In such studies, there is notable inter- and intra-species variation in the outcome between mice (Mus musculus) and rats (Rattus norvegicus). Among rats, the most reliable strains are Sprague-Dawley (SD) rats and spontaneously hypertensive rats (SHR) [33-36,38-41,43,46]. Both strains can manifest the many characteristics of MetS with no serious inconsistencies or conflicts. SD rats and SHR have been established by decades of research and are considered useful models of DIO [47-49]. However, the Wistar rat model has a recurring issue. Table 1 shows that the Wistar rat models demonstrate multiple reported complications in developing weight gain and hyperglycemia $[21,33,38,44,45]$. Wistar rats remain potential models, although not exclusively predisposed for DIO; perhaps they can be categorized towards a "generalized model". Only one study reported hyperglycemia and hypertriglyceridemia with abdominal fat mass of greater impact in Wistar rats as compared to SHR [36]. However, this may have been due to the shorter feeding period.

For mouse models, C57BL/6J mice are the M. musculus subjects of the highest frequency $[20,42]$. As per the test parameters, physiological and biological indicators do not pose issues for establishing MetS. One of the seven selected articles reported that C57BL/6 mice did not display weight gain and hyperglycemic levels, a reflection of Wistar rats. Astonishingly, C57BL/6J mice did not lead in a study on the genetic comparison of MetS in three different species. In fact, DBA/2 J (DBA) mice displayed a greater affinity for developing MetS over C57BL/6J mice [42]. However, strain specificity is not the sole factor, as the absence of weight gain and hyperglycemia levels may again be factored by the dosage and duration of the high-fructose diet, as discussed later. 


\subsubsection{Sex as a Limitation of MetS}

Table 1 shows that only a single study had included female subjects. The authors deduced that the presence of biological female organs and hormones plays an important role in protecting the body from MetS symptoms. Compared to non-ovariectomized female rats, OVX rats were at a relative disadvantage from the high-fructose diet, although still lacking in developing MetS compared to the male rats [34]. The outcome of that study only further implicates the use of male rats as sex-controlled models. The protective role of the ovarian hormone, estrogen, preserves the metabolic status of reproducing female individuals. The role of estrogen includes shedding excess adiposity, regulating insulin-mediated glucose and lipid metabolism, and reducing hyperphagia from behavioral changes and stress levels $[50,51]$. To strengthen this point, various studies on menopausal symptoms, where estrogen production is decreased, have noted the propensity of female subjects to accumulate abdominal fat mass, dyslipidemia, hyperglycemia, hypertension, and inflammation, the prime candidates for obesity and other health risks [51,52]. Hormone-driven satiety changes and increased dietary intake have also been reported from menopausal stages that mediate the development of these symptoms.

\subsection{Test Parameter Optimization and Selection}

\subsubsection{Physiological and Biochemical Parameters}

Referring to Table 1, the common physiological and biochemical parameters reflect the categories of symptoms established under the definition of MetS; they are: increased body weight (BW), hypertension, hyperglycemia, dyslipidemia, and insulin resistance [8]. The commonality of including caloric intake (hyperphagia) is subjective, as non-significant outcomes have been reported. The table shows that there were no correlations or patterns, but random chances when factored by fructose concentration and duration $[20,21,34,38,44-46]$. The same randomness has been observed in the outcomes for organ or tissue mass [21-23,33,34,36-39,42,44,45]. However, the collection of complete organs or specific tissue mass is highly encouraged for further analysis, as described below.

\subsubsection{Additional Parameters of MetS-Related Diseases}

Table 2 lists the objectives of the individual studies and their respective test parameters (as additional parameters). The purpose is to share the potential of several selected parameters that may be considered in the future for establishing and studying MetS models. Most MetS studies were not performed independently but were supplementary to other diseases. Two outstanding candidates are the inclusion of histological staining and inflammatory markers. The inflammatory markers that are significantly impacted and are useful indicators are: C-reactive protein [37], TNF- $\alpha$, IL-6 [20-23], IL-1 $\beta$ [22], and IL-10 [20,22]. Tables 1, 3 and 4 show that several hallmarks of MetS were not achieved, namely BW, hyperglycemia, and hypertriglyceridemia [21,33,38,44,45]. Through histological findings, these studies were able to identify significant changes at an anatomical level, even though visible but non-significant alterations were reported by the biochemical markers $[23,36,37,44]$. Signs such as liver fibrosis, abdominal fat deposition, and vascularization were clear. Despite its inclusion, histopathological analysis could only confirm the presence of changes, but was unable to quantify their severity. Incorporating higher-sensitivity analysis, for example, genetic and protein expression analysis, would refine the significance of the results. These methods would enable detailed quantitative and qualitative analysis of the imbalanced metabolites contributing to MetS [20,35,39,42,46]. In brief, complex analysis through advanced machination can provide precise diagnoses that may advance medical care towards personalized medication and novel therapeutics. 
Table 2. Additional biological parameters, methodology, and outcomes of MetS and related diseases.

\begin{tabular}{|c|c|c|c|}
\hline No. & $\begin{array}{l}\text { First author, Year of } \\
\text { Publication, and Main Study Objective } \\
\text { (Cardiac, Diabetes, Bone, Cancer) }\end{array}$ & $\begin{array}{c}\text { Additional Biological Parameters } \\
\text { (Inflammatory Markers, Genes, Proteins) }\end{array}$ & Outcomes of Additional Biological Parameters \\
\hline 1. & $\begin{array}{l}\text { Zemančiková et al. (2016) [33] } \\
\text { MetS and cardiovascular function }\end{array}$ & $\begin{array}{l}\text { Isometric tension endothelium-dependent } \\
\text { vasorelaxation, adrenergic contractions in } \\
\text { endothelium-intact mesenteric arteries }\end{array}$ & $\begin{array}{l}\text { Impaired relaxation of the aorta in both SHR and Wistar rats but not in nitric oxide } \\
\text { (NO)-deficient Wistar rats. Diminution of adrenergic contractions was observed in SHR } \\
\text { mesenteric arteries, but not in the Wistar rat counterparts. Contractile response in } \mathrm{K}^{+} \\
\text {concentrated bath solution was not altered between groups }\end{array}$ \\
\hline 2. & $\begin{array}{l}\text { Rattanavichit et al. (2016) [34] } \\
\text { MetS and ovarian hormone function }\end{array}$ & $\begin{array}{l}\text { Wet weight of uterus and skeletal muscle } \\
\text { (soleus). INS-mediated muscle GLU transport } \\
\text { activity. Analysis of signaling elements in } \\
\text { skeletal muscle (IRS-1 Tyr989, IRS-1 Ser307, } \\
\text { Akt Ser451, AS160 Ser588, JNK } \\
\text { Thr183/Tyr185, p38 MAPK Thr180/Tyr182) }\end{array}$ & $\begin{array}{l}\text { INS resistance was not induced in the skeletal muscles of non-OVX and OVX female rats, } \\
\text { while male rats exhibited the following changes: } \\
\text { - } \quad \text { Decreased -> IRS-1 Tyr989 (34\%), Akt Ser451 (30\%), AS160 Ser588 (43\%) } \\
\text { - Increased -> IRS-1 Ser307 (78\%), JNK Thr183/Tyr185 (69\%), p38 MAPK } \\
\text { Thr180/Tyr182 (81\%) } \\
\text { The above genetic analysis shows that male rats had significantly impaired INS-mediated } \\
\text { GLU uptake, leading to loss of skeletal muscle mass and mitochondrial activity in } \\
\text { energy 'production }\end{array}$ \\
\hline 3. & $\begin{array}{l}\text { Hsieh et al. (2016) [35] } \\
\text { MetS and liver function }\end{array}$ & $\begin{array}{l}\text { 2D gel-based proteomics. } \\
\text { MALDI-TOF/MS-MS to identify hepatic } \\
\text { protein expression patterns in rat liver }\end{array}$ & $\begin{array}{l}\text { Analysis identified proteins that were: } \\
\text { - Increased -> FAS, 78-kDa G-RP, LAT1, triokinase, GFAP, FBP-1, MAAI, GSTA3, PRDX1 } \\
\text { - } \quad \text { Decreased -> ACSM1, GDI-1 } \\
\text { These results indicate the impaired FA metabolism onset of carcinogenicity, inflammatory } \\
\text { mediators (FAS), and metabolic dysfunction originating from the liver }\end{array}$ \\
\hline 4. & $\begin{array}{l}\text { Lirio et al. (2016) [36] } \\
\text { MetS and NAFLD }\end{array}$ & Histology of liver tissue & There was interstitial fat deposition and fibrosis in the liver of SHR only \\
\hline 5. & $\begin{array}{l}\text { Bargut et al. (2017) [20] } \\
\text { MetS and WAT }\end{array}$ & $\begin{array}{l}\text { Histology of eWAT (adipocyte area), RT-qPCR, } \\
\text { Western blotting }\end{array}$ & $\begin{array}{l}\text { Hfru-treated rats displayed adipocyte area increase by } 21 \% \text {, implying hypertrophy, } \\
\text { inflammation, and uncontrolled lipolysis compared to control groups } \\
\text { Inflammatory markers: } \\
\text { - } \quad \text { Increased } \rightarrow \text { TNF- } \alpha, \text { IL-6, F4 } / 80, \mathrm{MCP} 1, \mathrm{pERK}, \mathrm{pJNK}, \mathrm{NF}-\mathrm{KB} \\
\text { - } \quad \text { Anti-inflammatory markers: } \\
\text { - } \quad \text { Increased } \rightarrow \text { ATGL, HSL, pHSL, } \beta 3-\mathrm{AR}, \mathrm{CD} 36, \mathrm{aP} 2 \\
\text { - } \quad \text { Decreased } \rightarrow \text { Adiponectin, PPAR- } \gamma \text { gene, perilipin, pAMPK, pAKT } \\
\text { - } \quad \text { No change }->\text { IL-10 gene and PPAR- } \gamma \text { protein }\end{array}$ \\
\hline
\end{tabular}


Table 2. Cont

\begin{tabular}{|c|c|c|}
\hline No. & $\begin{array}{l}\text { First author, Year of } \\
\text { Publication, and Main Study Objective } \\
\text { (Cardiac, Diabetes, Bone, Cancer) }\end{array}$ & $\begin{array}{c}\text { Additional Biological Parameters } \\
\text { (Inflammatory Markers, Genes, Proteins) }\end{array}$ \\
\hline 6. & $\begin{array}{l}\text { Bratoeva et al. (2018) [37] } \\
\text { MetS and renal function }\end{array}$ & Histological study of the kidney \\
\hline 7. & $\begin{array}{c}\text { Ramos et al. (2017) [38] } \\
\text { MetS }\end{array}$ & No additional parameters were observed \\
\hline 8. & $\begin{array}{l}\text { Abdelrahman et al. (2018) [21] } \\
\text { MetS and inflammatory and } \\
\text { oxidative markers }\end{array}$ & $\begin{array}{l}\text { Inflammatory markers, oxidative stress and } \\
\text { NO in fructose-fed rats: Aortic endothelin-1, } \\
\text { plasma and aortic NO, plasma TNF- } \alpha \text {, IL- } 6 \text {, } \\
\text { plasma superoxide dismutase (SOD), plasma } \\
\text { catalase, plasma MDA. Abdominal aorta } \\
\text { removed and weighed }\end{array}$ \\
\hline
\end{tabular}

9. $\quad$ Ng et al. (2018) [39] MetS and renal function

Chen et al. (2018) [40]

10. MetS and bladder function

Gambaro et al. (2018) [22] MetS and adipocyte function

$11 . \quad$ MetS and adipocyte function

Gene expression study and

immunohistochemistry of the liver and kidney

Rat bladder was processed into individual muscle strips for organ bath pharmacological studies. RT-PCR and Western blot analysis of

bladder control via genetic and protein expressions of cannabinoid receptor (CB) $1 / \mathrm{CB} 2$ receptors
Metabolic mRNA analysis of adipose tissue. Histological analysis of adipose tissue

\section{Outcomes of Additional Biological Parameters}

Proximal and distal section of the tubular region revealed signs of vacuolization and degeneration, respectively. Vascular rupture, scarring, and atherosclerotic manifestations were apparent, but not in the control. The glomeruli and subendothelial layers of blood vessels had nodule formation, shown by positive amyloid stains. These traits show the loss of glomeruli function and vascular deterioration that matched MetS symptoms

\section{No additional findings were made}

When compared to the control, the Hfru group displayed significant levels of:

- $\quad$ Increased $\rightarrow$ MDA, TNF- $\alpha$, IL-6, aortic NO, endothelin-1

- $\quad$ Reduced $\rightarrow$ Catalase, SOD

These results complement the biochemical parameters that had established progressive inflammation, oxidative damage, and hypertensive disorder

The FR-5 group expressed lower GLUT1, compensated by increased GLUT2 and GLUT9 compared to the FR-3 group. Both groups had equally increased SGLT1, SGLT2, renal UA expression, and FA deposition. Increased GLUT9 and UA are correlated to signs of hyperuricemia; SGLT1:SGLT2 imbalance is identified as ineffective sodium-glucose reabsorption function of the kidney. These findings also describe the loss of INS function compensated by SGLT2 INS-independent function of GLU reabsorption

Acetylcholine bladder muscle strip contraction showed statistically insignificant differences between Hfru and control groups. Western blotting showed decreased CB1/CB2 protein levels following MetS diet induction. Similarly, RT-PCR showed that CB1/CB2 mRNA was decreased significantly in the fructose group. These outcomes signify the loss of the homeostatic role of CB1 and CB2, affecting the parasympathetic stimulus for bladder function, as observed in the MetS model

In both periods ( 6 and 10 weeks), there was increased eWAT; decreased adiponectin, IL-6, IL-10, TNF- $\alpha$, CD206; and hypertrophy and parenchymatous degeneration of hepatocytes. Only the 10-week group had decreased Il-10 and Il-1b, with instances of lipid accumulation foci in histological sections compared to the 6-week group. These findings show that the accumulation of fat mass increased proportionally to the feeding duration. It stimulated inflammatory macrophages (M1) and decreased the anti-inflammatory response, leading to liver damage and loss of function in the diet-induced MetS model 
Table 2. Cont.

No. Publication, and Main Study Objective (Cardiac, Diabetes, Bone, Cancer)

2. Subramani et al. (2019) [41] MetS

Fakhoury-Sayegh et al. (2019) [23] MetS with hepatic, renal, and pancreatic function
Additional Biological Parameters (Inflammatory Markers, Genes, Proteins)
Outcomes of Additional Biological Parameters

The Hfru group had significantly increased TBARS and decreased SOD but non-significantly increased NF- $\mathrm{KB}$ compared to the control diet groups. The Hfru group

Oxidative stress measurement (TBARS, SOD). Rat nuclear factor kappa B (NF-kB).

Histopathology of the liver and heart hypertrophic heart to circulate and compensate for atherosclerosis and other loss of
Oxidative stress (MDA). Serum adiponectin levels. Inflammatory markers (TNF- $\alpha$ and IL-6). Histopathology examinations of the liver, kidney, pancreas, and epididymal fat pads had fatty infiltration, with micro- and macrovesicular hepatic steatosis and hypertrophied heart compared to the control groups. These results show the effect of MetS that leads to a function. Fructose feeding accumulated fatty deposition and ROS and inflammatory cytokine secretion, leading to dyslipidemia of the liver

Adiponectin levels decreased in group 2 and 3 (20\% and 30\% Hfru, respectively). MDA levels decreased in all groups except group 2. TGL, TNF- $\alpha$, and IL- 6 were not significant between the fructose groups and control after 16 weeks. The control and group 2 and 3 had minor signs of microvesicular steatosis. Only group 4 (30\% Hfru) presented milder microvacuolar steatosis. Necroinflammation and fibrosis were absent from all groups, but perisinusoidal fibrosis was apparent $(>20 \%)$ in group 3 and 4 . Group 3 and 4 kidneys showed mild renal inflammation $(>60 \%)$ and lower glomerulosclerosis and interstitial renal fibrosis. The pancreas did not show hyperplasia or hypertrophy of the islets of Langerhans. Islet distribution, size, and shape were not altered or fibrotic. After 16-week Hfru intervention, the onset of liver fibrosis and kidney failure were proportional to Hfru dosage. INS function and histopathology of the pancreas were unchanged, thus remaining functional

Strain-specific DEGs were discovered, as DBA mice represented the largest volumes in all three tissues for lipid metabolism. However, some overlapping DEGs did not show strain specificity but were affected by fructose metabolism. The categories were:

RNA sequencing of the hypothalamus, liver, and mWAT to determine the key drivers in liver function to validate strain individuality properties. Subsequently, deduce differentially expressed genes (DEGs) in the hypothalamus, liver, and WATs
1. Xenobiotic stimulus (Gstp1, Ephx1, Gstm1)

2. Organic cyclic compounds (Abca1, Id3, Abat)

3. Metabolic processes (Abca1, Htatip2, Grhpr, Nudt7)

4. Transcriptional regulation (Ier5, Jun, Id3)

5. Immune modulation $(C d 200)$

15. Mustafa et al. (2020) [43]
MetS $\quad$ No additional parameters were observed $\quad$ No additional findings were made


Table 2. Cont.

First author, Year of

No. Publication, and Main Study Objective

(Cardiac, Diabetes, Bone, Cancer)

Ferreira-Santos et al. (2020) [44]

MetS
Additional Biological Parameters (Inflammatory Markers, Genes, Proteins)
Outcomes of Additional Biological Parameters

$\mathrm{LVH}$, liver index, oxidative stress, and adiposity increased significantly in the fructose group compared to the control. Histological staining showed morphological alterations in hepatocytes and adipocytes. The liver of fructose-fed rats had lipid accumulation. Endothelial dysfunction manifested from the reduced relaxation in response to acetylcholine (Ach) in the aortic and mesenteric rings of the fructose group. A Hfru diet facilitated liver steatosis and hypertension due to stiff endothelial contraction and lipid-derived oxidative damage to metabolic organs

Liver, kidney, heart, and SM vitamin E content were significantly higher in the fructose group than in the control by 2 weeks. However, vitamin E in WAT was significantly lower in the fructose group, which continued on a downward trend until 6 weeks. Heart, WAT, and liver LPO increased and achieved statistical significance by 6 weeks. Vitamin E plays a pivotal role in antioxidative responses, which was hampered by MetS induced by a Hfru diet. This accrued damage in the heart, liver, and organs surrounded by WAT

Serum levels of renin and Ang II were significantly elevated by fructose supplementation. Fructose intake increased expression of Agt in the liver and Ace in the lungs. Fructose intake increased AT1R and Ang I protein levels in the kidney. Histological examination showed no significant effect on collagen deposition and fibrosis, which would have appeared blue upon trichrome staining. The fluctuation of the adrenal metabolites above affected the lung, kidney, and liver functions. Hence, the fructose diet disrupted pulmonary, hepatic, and renal function by altering the adrenal maintenance of vascular properties crucial for biochemical homeostasis and nutrient supplementation 
Table 3. Durations of MetS induction to physiological and biochemical outcomes of MetS.

\begin{tabular}{|c|c|c|c|c|c|c|c|c|}
\hline \multirow[b]{2}{*}{$\begin{array}{l}\text { Duration of MetS } \\
\text { Induction (weeks) }\end{array}$} & \multirow[b]{2}{*}{$\begin{array}{l}\text { References of } \\
\text { Study(s) }\end{array}$} & \multicolumn{7}{|c|}{ Physiological and Biochemical Parameters of MetS } \\
\hline & & $\begin{array}{c}\text { Increased BW, } \\
\text { Length or } \\
\text { Abdominal } \\
\text { Circumference }\end{array}$ & $\begin{array}{c}\text { Increased } \\
\text { Organ or } \\
\text { Tissue Mass }\end{array}$ & $\begin{array}{c}\text { Increased Caloric } \\
\text { Intake } \\
\text { (Hyperphagia, } \\
\text { Hyperleptinemia) }\end{array}$ & $\begin{array}{c}\text { High Blood } \\
\text { Pressure } \\
\text { (Hypertension) }\end{array}$ & $\begin{array}{c}\text { High Blood } \\
\text { Glucose Levels } \\
\text { (Hyperglycemia) }\end{array}$ & $\begin{array}{c}\text { Dyslipidemia } \\
\text { (Hypertriglyc- } \\
\text { eridemia, } \\
\text { Hypercholes- } \\
\text { terolemia) }\end{array}$ & $\begin{array}{l}\text { Hyperinsulinemia } \\
\text { or Insulin } \\
\text { Resistance }\end{array}$ \\
\hline 2 & {$[45,46]$} & / & / & $\mathrm{X}$ & $\mathrm{O}$ & $\mathrm{O}$ & $\mathrm{O}$ & / \\
\hline 3 & {$[20]$} & $X$ & $X$ & $\mathrm{O}$ & / & $\mathrm{O}$ & / & / \\
\hline 4 & {$[38,45]$} & I & $\mathrm{O}$ & $\mathrm{O}$ & $\mathrm{O}$ & $\mathrm{O}$ & / & 1 \\
\hline 5 & {$[21]$} & $x$ & $\mathrm{O}$ & $\mathrm{O}$ & / & $\mathrm{O}$ & / & 1 \\
\hline 6 & {$[22,34,36,41,43,45]$} & / & / & / & / & / & / & / \\
\hline 8 & {$[33,38]$} & / & / & $\mathrm{O}$ & / & / & / & / \\
\hline 10 & [22] & / & $\mathrm{O}$ & $\mathrm{O}$ & $\mathrm{O}$ & $X$ & / & / \\
\hline 12 & {$[38,39,42,44]$} & 1 & / & $X$ & $\mathrm{O}$ & $x$ & $\mathrm{O}$ & / \\
\hline 13 & [39] & / & $\mathrm{O}$ & $\mathrm{O}$ & / & $\mathrm{O}$ & / & $\mathrm{O}$ \\
\hline 16 & {$[23,35,37]$} & $X$ & $X$ & $X$ & / & / & / & / \\
\hline 21 & [39] & / & / & $\mathrm{O}$ & $\mathrm{O}$ & $x$ & / & $\mathrm{O}$ \\
\hline
\end{tabular}

$(/)=$ statistically significant results, $(p<0.05) ;(X)=$ statistically non-significant results $(p>0.05) ;(\mathrm{O})=$ test parameter unavailable or not assessed in study $(\mathrm{s})$

Table 4. Concentration of types of fructose diet to physiological and biochemical outcomes of MetS.

\begin{tabular}{|c|c|c|c|c|c|c|c|c|c|}
\hline \multirow[b]{2}{*}{$\begin{array}{c}\text { Conc. of } \\
\text { Fructose (\%) }\end{array}$} & \multicolumn{2}{|c|}{ References of Study(s) } & \multicolumn{7}{|c|}{ Physiological and Biochemical Parameters of MetS } \\
\hline & Solution & Pellet & $\begin{array}{c}\text { Increased BW, } \\
\text { Length or } \\
\text { Abdominal } \\
\text { Circumference }\end{array}$ & $\begin{array}{c}\text { Increased } \\
\text { Organ or } \\
\text { Tissue } \\
\text { Mass }\end{array}$ & $\begin{array}{c}\text { Increased Caloric } \\
\text { Intake } \\
\text { (Hyperphagia, } \\
\text { Hyperleptinemia) }\end{array}$ & $\begin{array}{c}\text { High Blood } \\
\text { Pressure } \\
\text { (Hypertension) }\end{array}$ & $\begin{array}{c}\text { High Blood } \\
\text { Glucose } \\
\text { Levels } \\
\text { (Hyperglycemia) }\end{array}$ & $\begin{array}{c}\text { Dyslipidemia } \\
\text { (Hypertriglyc- } \\
\text { eridemia, } \\
\text { Hypercholes- } \\
\text { terolemia) }\end{array}$ & $\begin{array}{l}\text { Hyperinsulinemia } \\
\text { or Insulin } \\
\text { Resistance }\end{array}$ \\
\hline 8 & [42] & - & / & / & $X$ & $\mathrm{O}$ & $X$ & $\mathrm{O}$ & $\mathrm{O}$ \\
\hline 10 & {$[33,34,36]$} & [23] & 1 & $\mathrm{O}$ & I & / & $\mathrm{O}$ & / & / \\
\hline 20 & {$[22,38]$} & {$[23]$} & 1 & / & $x$ & $\mathrm{O}$ & $\mathrm{O}$ & 1 & $\mathrm{O}$ \\
\hline 30 & {$[-$} & {$[23]$} & $x$ & $x$ & $x$ & $\mathrm{O}$ & 1 & 1 & / \\
\hline 45.43 & - & [20] & $x$ & $x$ & $\mathrm{O}$ & / & $\mathrm{O}$ & / & / \\
\hline 60 & - & {$[21,35,39-41,45]$} & $x$ & / & / & 1 & / & / & / \\
\hline 66 & - & [41] & / & $\mathrm{O}$ & $\mathrm{O}$ & / & / & / & $\mathrm{O}$ \\
\hline
\end{tabular}

$(/)=$ statistically significant results, $(p<0.05) ;(\mathrm{X})=$ statistically non-significant results $(p>0.05) ;(\mathrm{O})=$ test parameter unavailable or not assessed in study $(\mathrm{s})$. 


\subsection{Duration and Dosage of High-Fructose Diet}

\subsubsection{Period of MetS Induction in Animal Models}

The 6-week duration was the protocol duration with the greatest frequency in studies on MetS induction (Table 3). Additionally, the outcomes of the six 6-week studies achieved all categories of the MetS physiological and biochemical parameters listed in Table $1[22,34,36,41,43,45]$. Logically, prolonged induction of a MetS diet would have common outcomes, as it is described as a chronic induced syndrome, deteriorating over time. However, Tables 1 and 3 show that prolonged duration does not achieve several factors such as body mass, organ or tissue mass, and hyperglycemia. Another confounding variable is that species, sex, and fructose diet dosage were not considered. Upon cross-factoring, the outcomes of these three factors were identical to that of studies discussing species differences, specifically Wistar rats $[21,33,38,44,45]$. This highlights the importance of strain selection and the predisposition of the strains to diet-induced obesity and MetS symptoms.

\subsubsection{Concentration of Fructose Diet for Inducing MetS}

A high-fructose diet is a part of the triad that includes species and duration for inducing MetS. The selection of fructose concentration is often an overlooked preparation, as intake in rats relies more on palatability and ease of access over satiation levels $[53,54]$. These special diets are noticeably difficult to solidify and may be unappealing to the rats if produced in private or non-commercially. However, fructose dissolved in water did not pose difficulties, as hydration has greater importance over hunger. The natural ability of rats to resist hunger and persist in harsh environments differs from that of humans and may be a confounding variable by limiting the induction of MetS via DIO [9,54]. Table 4 shows that the most reliable concentrations for solution- and pellet-based high-fructose diets were $40 \%$ and $66 \%$, respectively [41,43]. These two studies were able to achieve all MetS determinant parameters, although they did not incorporate all aspects thereof, such as organ or tissue mass, caloric intake, blood glucose levels, and insulin resistance. However, Table 1 shows that a $60 \%$ fructose-based diet encompassed all categories of MetS and achieved all but one: increased BW, length, or abdominal circumference [21,35,39,40,45]. Of those five articles, only one reported visibly increased BW, but it was not statistically significant [35]. However, this challenge from the individual study could be subjective; hence, $60 \%$ fructose pellets might be deemed equally competent to the single-study $66 \%$ fructose pellets [41]. Arguably, another factor to consider is the price of pellets, which is much higher than that of crystalline fructose. These special diet pellets can be made with economically viable ingredients, although this may require further optimization, while purchasing commercial diets may not be a sustainable cost for long-term studies. Crystalline fructose in water is, however, an inexpensive and manageable source.

This review was performed emphasizing a fructose diet exerting physiological and biochemical changes that lead to MetS manifestation. An abundance of experimental protocols utilize high-carbohydrate diet, high-fat diet, and sucrose water (or a combination thereof). Whether these are superior to a pure-fructose diet has not been reviewed here and may serve as an alternative. Carvajal et al. reviewed different diets for inducing MetS in 2020 [9]. Their review could serve as a reference for analyzing the nutritional properties of single or combination high-fat, -sugar, and -salt pellets. Other aspects have not been discussed, such as MetS models in other species. Although rodents are extensively used in scientific research, it is likely due to less restrictive ethics approval. Other mammalian models, such as felines, canines, leporids, swine, equines, and hominoids, may not benefit from this review. Hence, we suggest selecting from the available animal models before proceeding to protocol induction and optimization.

\section{Conclusions}

The best represented rodent species are SD rats and/or SHR and C57BL/6J mice. Ideally, the rodent subjects should be male to exclude the protective nature of female hormones against MetS development, or sex may otherwise be justified in female-specific 
studies. A study duration of $\geq 6$ weeks is optimal. The fructose concentration is dictated by the method of feeding, which varies with the researcher's ability to produce or procure pellets. Otherwise, $40 \%$ fructose water or $60 \%$ fructose pellets yield the best outcomes. Lastly, MetS is often studied in parallel or related to selected diseases. General parameters should encompass MetS prognosis of obesity, hypertension, hyperglycemia, hypertriglyceridemia, dyslipidemia, and insulin resistance. Here, we have tabulated the specific study parameters factored by MetS, and these parameters should be considered when planning studies involving MetS.

Author Contributions: Conceptualization, A.M.L.C., A.M.H.N. and Y.L.; Methodology, A.M.L.C. and Y.L.; Validation, A.M.L.C. and Y.L.; Formal Analysis, A.M.L.C.; Investigation, A.M.L.C.; Data Curation, A.M.L.C. \& Y.L.; Writing-Original Draft Preparation, A.M.L.C.; Writing-Review \& Editing, all authors. Visualization, all authors. Supervision, Y.L., M.H.M.Y. and A.M.H.N.; Project Administration, Y.L.; Funding Acquisition, Y.L. and A.M.H.N. All authors have read and agreed to the published version of the manuscript.

Funding: This work was supported by grants by Ming Medical Sdn. Bhd. (FF-2020-469) and Universiti Kebangsaan Malaysia (FF-2020-469/1 and DPK-2021-006).

Institutional Review Board Statement: Not applicable.

Informed Consent Statement: Not applicable.

Data Availability Statement: Not applicable.

Conflicts of Interest: The authors declare no conflict of interest. The granting agencies played no role in activities pertaining to the execution of the study and the submission of the manuscript.

\section{References}

1. Vague, J.; Jouve, A.; Delaage, M.; Teitelbaum, M. Les Relations de L'obesite et de L'arteriosclerose; Documentation scientifique Guigoz: Basel, Germany, 1958; Volume 41, pp. 1-20.

2. Haller, H.; Hanefeld, M. Synoptische Betrachtung Metabolischer Risikofaktoren. In Lipidstoffwechselstörungen; Haller, H., Hanefeld, M., Jaross, W., Eds.; Gustav Fischer Verlag: Jena, Germany, 1975; pp. 254-264.

3. World Health Organization: Obesity and Overweight. April 2020. Available online: https://www.who.int/news-room/factsheets/detail/obesity-and-overweight (accessed on 18 January 2021).

4. International Diabetes Federation: Diabetes Facts and Figures. 2 February 2020. Available online: https://www.idf.org/ aboutdiabetes/what-is-diabetes / facts-figures.html (accessed on 18 January 2021).

5. Li, Y.; Zhao, L.; Yu, D.; Wang, Z.; Ding, G. MetS prevalence and its risk factors among adults in China: A nationally representative cross-sectional study. PLOS ONE 2018, 13, e0199293.

6. Cordain, L.; Eaton, S.B.; Sebastian, A.; Mann, N.; Lindeberg, S.; Watkins, B.A.; O'Keefe, J.H.; Brand-Miller, J. Origins and evolution of the Western diet: Health implications for the 21st century. Am. J. Clin. Nutr. 2005, 81, 341-354. [CrossRef] [PubMed]

7. Roche, H.M.; Phillips, C.; Gibney, M.J. The MetS: The crossroads of diet and genetics. Proc. Nutr. Soc. 2005, 64, 371-377. [CrossRef]

8. Punthakee, Z.; Goldenberg, R.; Katz, P. Definition, Classification and Diagnosis of Diabetes, Prediabetes and MetS. Can. J. Diabetes 2018, 42, S10-S15. [CrossRef]

9. Carvajal, K.; Rodríguez-Correa, E.; González-Pérez, I.; Clavel-Pérez, P.I.; Contreras-Vargas, Y. Biochemical and nutritional overview of diet-induced MetS models in rats: What is the best choice? Nutr. Diabetes 2020, 10, 24.

10. Fontvieille, A.M.; Faurion, A.; Helal, I.; Rizkalla, S.W.; Falgon, S.; Letanoux, M.; Slama, G. Relative Sweetness of Fructose Compared With Sucrose in Healthy and Diabetic Subjects. Diabetes Care 1989, 12, 481-486. [CrossRef]

11. Bantle, J.P. Dietary Fructose and MetS and Diabetes. J. Nutr. 2009, 139, 1263S-1268S. [CrossRef] [PubMed]

12. Zargaraan, A.; Kamaliroosta, L.; Seyed Yagoubi, A.; Mirmoghtadaie, L. Effect of Substitution of Sugar by High Fructose Corn Syrup on the Physicochemical Properties of Bakery and Dairy Products: A Review. Nutr. Food Sci. Res. 2016, 3, 3-11. [CrossRef]

13. Avena, N.M.; Rada, P.; Hoebel, B.G. Evidence for sugar addiction: Behavioral and neurochemical effects of intermittent, excessive sugar intake. Neurosci. Biobehav. Rev. 2008, 32, 20-39. [CrossRef] [PubMed]

14. Hannou, S.A.; Haslam, D.E.; McKeown, N.M.; Herman, M.A. Fructose metabolism and metabolic disease. J. Clin. Investig. 2018, 128, 545-555. [CrossRef]

15. Kolderup, A.; Svihus, B. Fructose Metabolism and Relation to Atherosclerosis, Type 2 Diabetes, and Obesity. J. Nutr. Metab. 2015, 2015, 823081. [CrossRef] [PubMed]

16. Tappy, L.; Rosset, R. Health outcomes of a high fructose intake: The importance of physical activity. J. Physiol. 2019, 597, 3561-3571. [CrossRef] [PubMed]

17. Sanders, F.W.B.; Griffin, J.L. De novolipogenesis in the liver in health and disease: More than just a shunting yard for glucose. Biol. Rev. 2019, 91, 452-468. [CrossRef] [PubMed] 
18. Herman, M.A.; Samuel, V.T. The Sweet Path to Metabolic Demise: Fructose and Lipid Synthesis. Trends Endocrinol. Metab. 2016, 27, 719-730. [CrossRef]

19. Rizkalla, S.W. Health implications of fructose consumption: A review of recent data. Nutr. Metab. 2010, 7, 82. [CrossRef]

20. Bargut, T.C.L.; Santos, L.P.; Machado, D.G.L.; Aguila, M.B.; Mandarim-de-Lacerda, C.A. Eicosapentaenoic acid (EPA) vs. Docosahexaenoic acid (DHA): Effects in epididymal white adipose tissue of mice fed a high-fructose diet. Prostaglandins Leukot. Essent. Fat. Acids (PLEFA) 2017, 123, 14-24. [CrossRef]

21. Abdelrahman, A.M.; Al-Suleimani, Y.M.; Ashique, M.; Manoj, P.; Ali, B.H. Effect of infliximab and tocilizumab on fructose-induced hyperinsulinemia and hypertension in rats. Biomed. Pharmacother. 2018, 105, 182-186. [CrossRef]

22. Gambaro, S.; Zubiria, M.; Portales, A.; Rey, M.; Rumbo, M.; Giovambattista, A. M1 macrophage subtypes activation and adipocyte dysfunction worsen during prolonged consumption of a fructose rich diet. J. Nutr. Biochem. 2018, 61, 173-182. [CrossRef]

23. Fakhoury-Sayegh, N.; Trak-Smayra, V.; Sayegh, R.; Haidar, F.; Obeid, O.; Asmar, S.; Khazzaka, A. Fructose threshold for inducing organ damage in a rat model of non-alcoholic fatty liver disease. Nutr. Res. 2018, 62, 101-112. [CrossRef]

24. Zhou, J.; Zhang, Q.; Yuan, X.; Wang, J.; Li, C.; Sheng, H.; Qu, S.; Li, H. Association between Metabolic Syndrome and osteoporosis: A meta-analysis. Bone 2013, 57, 30-35. [CrossRef]

25. Wong, S.K.; Chin, K.Y.; Suhaimi, F.H.; Ahmad, F.; Ima-Nirwana, S. Effects of Metabolic Syndrome on bone mineral density, histomorphometry and remodelling markers in male rats. PLoS ONE 2018, 13, e0192416. [CrossRef] [PubMed]

26. Wong, S.K.; Chin, K.Y.; Suhaimi, F.H.; Ahmad, F.; Ima-Nirwana, S. The Relationship between Metabolic Syndrome and Osteoporosis: A Review. Nutrients 2016, 8, 347. [CrossRef]

27. Chin, K.Y.; Chan, C.Y.; Subramaniam, S.; Muhammad, N.; Fairus, A.; Ng, P.Y.; Jamil, N.A.; Aziz, N.A.; Ima-Nirwana, S.; Mohamed, N. Positive association between Metabolic Syndrome and bone mineral density among Malaysians. Int. J. Med. Sci. 2020, 17, 2585-2593. [CrossRef]

28. Chow, Y.Y.; Chin, K.Y. The Role of Inflammation in the Pathogenesis of Osteoarthritis. Mediat. Inflamm. 2020, $2020,8293921$. [CrossRef]

29. Nur-Vaizura, M.; Soelaiman, I.M.; Chin, K.Y. Are oxidative stress and inflammation mediators of bone loss due to estrogen deficiency? A review of current evidence. Endocr. Metab. Immune Disord. Drug Targets 2020, 20, 1478-1487.

30. Hoyer, S. The aging brain. Changes in the neuronal insulin/insulin receptor signal transduction cascade trigger late-onset sporadic Alzheimer disease (SAD). A Mini-Review. J. Neural Transm. 2002, 109, 991-1002. [CrossRef]

31. Moreira, P.I.; Santos, M.S.; Seiça, R.; Oliveira, C.R. Brain mitochondrial dysfunction as a link between Alzheimer's disease and diabetes. J. Neurol. Sci. 2007, 257, 206-214. [CrossRef] [PubMed]

32. Zhou, C.; Huang, Y.; Przedborski, S. Oxidative Stress in Parkinson's Disease. Ann. N. Y. Acad. Sci. 2008, 1147, 93-104. [CrossRef] [PubMed]

33. Zemančiková, A.; Torok, J. Comparison of high fructose-induced cardiometabolic impairments in two different rat hypertensive models. Curr. Top. Toxicol. 2016, 12, 25-32.

34. Rattanavichit, Y.; Chukijrungroat, N.; Saengsirisuwan, V. Sex differences in the metabolic dysfunction and insulin resistance of skeletal muscle glucose transport following high fructose ingestion. Am. J. Physiol. -Regul. Integr. Comp. Physiol. 2016, 311, R1200-R1212. [CrossRef]

35. Hsieh, C.C.; Liao, C.C.; Liao, Y.C.; Hwang, L.S.; Wu, L.Y.; Hsieh, S.C. Proteomic changes associated with Metabolic Syndromein a fructose-fed rat model. J. Food Drug Anal. 2016, 24, 754-761. [CrossRef] [PubMed]

36. Lírio, L.M.; Forechi, L.; Zanardo, T.C.; Batista, H.M.; Meira, E.F.; Nogueira, B.V.; Mill, J.G.; Baldo, M.P. Chronic fructose intake accelerates non-alcoholic fatty liver disease in the presence of essential hypertension. J. Diabetes Its Complicat. 2016, 30, 85-92. [CrossRef]

37. Bratoeva, K.; Nikolova, S.; Merdzhanova, A.; Stoyanov, G.S.; Dimitrova, E.; Kashlov, J.; Radanova, M. Association between Serum CK-18 Levels and the Degree of Liver Damage in Fructose-Induced Metabolic Syndrome. Metab. Syndr. Relat. Disord. 2018, 16, 350-357. [CrossRef] [PubMed]

38. Ramos, V.W.; Batista, L.O.; Albuquerque, K.T. Effects of fructose consumption on food intake and biochemical and body parameters in Wistar rats. Efeitos do consumo de frutose sobre ingestão alimentar, parâmetros bioquímicos e corporais em ratos Wistar. Revista portuguesa de cardiologia: Orgao oficial da Sociedade Portuguesa de Cardiologia = Portuguese journal of cardiology: An official journal of the Portuguese Society of Cardiology. Rev. Port Cardiol. 2017, 36, 937-941.

39. Ng, H.Y.; Lee, Y.T.; Kuo, W.H.; Huang, P.C.; Lee, W.C.; Lee, C.T. Alterations of Renal Epithelial Glucose and Uric Acid Transporters in Fructose Induced Metabolic Syndrome. Kidney Blood Press. Res. 2018, 43, 1822-1831. [CrossRef]

40. Chen, I.H.; Cheng, J.T.; Tong, Y.C. Metabolic Syndrome Induced Bladder Cannabinoid Receptor Changes in the Fructose-Fed Rats. LUTS Low. Urin. Tract Symptoms 2017, 10, 198-203. [CrossRef]

41. Subramani, B.; Krishnamurthy, B. Effects of Momordica Charantia (Bitter gourd) on Oxidative Stress and Pro-Inflammatory Marker in Metabolic Syndrome Using a High-Fructose Diet Induced Rat Model. Biomed. Pharmacol. J. 2019, 11, 305-324. [CrossRef]

42. Zhang, G.; Byun, H.R.; Ying, Z.; Blencowe, M.; Zhao, Y.; Hong, J.; Shu, L.; Krishnan, K.C.; Gomez-Pinilla, F.; Yang, X. Differential metabolic and multi-tissue Transcriptomic responses to fructose consumption among genetically diverse mice. Biochim. Biophys. Acta (BBA) Mol. Basis Dis. 2019, 1866, 165569. [CrossRef]

43. Mustafa, N.G.; Hasan, M.K. Biochemical investigation of an experimentally induced Metabolic Syndrome in rats. Indian J. Anim. Res. 2020, 52, 168-172. [CrossRef] 
44. Ferreira-Santos, P.; Aparicio, R.; Carrón, R.; Montero, M.J.; Sevilla, M.Á. Lycopene-supplemented diet ameliorates MetS induced by fructose in rats. J. Funct. Foods 2020, 73, 104098. [CrossRef]

45. Kitagawa, A.; Ohta, Y.; Ohashi, K.; Yashiro, K.; Fukuzawa, K. Effect of High Fructose-Induced Metabolic Syndrome on Tissue Vitamin E and Lipid Peroxide Levels in Rats. J. Nutr. Sci. Vitaminol. 2020, 66, 200-206. [CrossRef]

46. Kim, M.; Do, G.Y.; Kim, I. Activation of renin-angiotensin system in high fructose-induced Metabolic Syndrome. Korean J. Physiol. Pharmacol. 2020, 24, 319-328. [CrossRef]

47. Marques, C.; Meireles, M.; Norberto, S.; Leite, J.; Freitas, J.; Pestana, D.; Faria, A.; Calhau, C. High-fat diet-induced obesity Rat model: A comparison between Wistar and Sprague Dawley Rat. Adipocyte 2015, 5, 11-21. [CrossRef] [PubMed]

48. Giles, E.D.; Jackman, M.R.; MacLean, P.S. Modeling Diet-Induced Obesity with Obesity-Prone Rats: Implications for Studies in Females. Front. Nutr. 2016, 3, 50. [CrossRef] [PubMed]

49. Rojas, J.M.; Bolze, F.; Thorup, I.; Nowak, J.; Dalsgaard, C.M.; Skydsgaard, M.; Berthelsen, L.O.; Keane, K.A.; Søeborg, H.; Sjögren, I.; et al. The Effect of Diet-induced Obesity on Toxicological Parameters in the Polygenic Sprague Dawley Rat Model. Toxicol. Pathol. 2018. [CrossRef] [PubMed]

50. Leeners, B.; Geary, N.; Tobler, P.N.; Asarian, L. Ovarian hormones and obesity. Hum. Reprod. Update 2017, 23, 300-321. [CrossRef] [PubMed]

51. Zhu, J.; Ji, M.; Xing, L.; Yu, Z.; Guo, X.; Chen, X.; Shu, J. Ovarian Hormonal Change-Related Energy Metabolism and Obesity in Menopausal Women. Horm. Ther. Replace. Cancer Aging-Relat. Dis. 2020. [CrossRef]

52. Boonyaratanakornkit, V.; Pateetin, P. The Role of Ovarian Sex Steroids in Metabolic Homeostasis, Obesity, and Postmenopausal Breast Cancer: Molecular Mechanisms and Therapeutic Implications. BioMed Res. Int. 2015, 2015, 140196. [CrossRef] [PubMed]

53. Toth, L.A.; Gardiner, T.W. Food and water restriction protocols: Physiological and behavioral considerations. Contemp. Top. Lab. Anim. Sci. 2020, 39, 9-17.

54. Corwin, R.L.; Avena, N.M.; Boggiano, M.M. Feeding and reward: Perspectives from three rat models of binge eating. Physiol. Behav. 2011, 104, 87-97. [CrossRef] 